\title{
Preparation of a New Polymeric Deep Eutectic Solvent and Its Application in Vortex-Assisted Liquid-Liquid Microextraction of Parabens in Foods, Cosmetics and Pharmaceutical Products
}

\author{
Dandan Ge, ${ }^{\circledR *, a}$ Ying Gao, ${ }^{a}$ Yunxin Cao, ${ }^{a}$ Enrui Dai ${ }^{a}$ and Lin Yuan ${ }^{a}$ \\ ${ }^{a}$ School of Chemistry and Chemical Engineering, Kunming University, 650214 Kunming, China
}

\begin{abstract}
A vortex-assisted liquid-liquid microextraction method combined with high performance liquid chromatography-diode array detector has been developed for the determination of parabens in foods, cosmetics and pharmaceutical products. In this work, polymeric deep eutectic solvents composed of DL-menthol and polyethylene glycol with three different light grades were prepared successfully for the first time and used as extraction solvents. The influencing parameters on the extraction efficiency including type of extraction solvent, salt addition, composition of deep eutectic solvent, volume of extraction solvent, extraction time and solution $\mathrm{pH}$ were evaluated. Under the optimized conditions, using DL-menthol and polyethylene glycol 400 at a molar ratio of $1: 1$ as the extraction solvent, the method exhibited good linearity with linear coefficients greater than 0.9995 . Limits of detection and limits of quantification were in the range of $0.3-2 \mathrm{ng} \mathrm{mL}^{-1}$ and 1-5 $\mathrm{ng} \mathrm{mL}^{-1}$, respectively. The proposed method was successfully applied to determine four parabens in seven commercial products. The proposed method is simple, green and efficient, and could be applied to determine parabens in complex matrices.
\end{abstract}

Keywords: deep eutectic solvent, vortex-assisted dispersive liquid-liquid microextraction, parabens, high performance liquid chromatography

\section{Introduction}

Parabens are commonly used as preservatives to prevent the development of undesirable microorganisms in food products, cosmetics, personal care and pharmaceutical products. ${ }^{1,2}$ However, various studies ${ }^{3-5}$ have reported that parabens may pose a potential hazard to human health, being related to the carcinogenic and estrogenic threats. In view of this, a maximum content of $0.4 \%(\mathrm{~m} / \mathrm{m})$ for an individual paraben and a limit of $0.8 \%(\mathrm{~m} / \mathrm{m})$ for total parabens in commercial products have been regulated by European Union. ${ }^{6}$

To determine the contents of parabens in complex sample matrices, sample preparation techniques are required prior to chromatographic or hyphenated analysis. In terms of clean-up and preconcentration process, the removal of impurities and enrichment of analytes is challenging due to the complex matrices involved..$^{7,8}$ For the extraction of parabens from different samples, minimized liquid-liquid extraction methods including single-drop microextraction, ${ }^{9}$ salt-assisted cloud point

*e-mail: dandangeiris@163.com extraction, ${ }^{1}$ stirring-assisted drop-breakup microextraction ${ }^{10}$ and dispersive liquid-liquid microextraction (DLLME) ${ }^{11,12}$ have been reported. DLLME, as a powerful microextraction technique, was introduced by Rezaee et al. ${ }^{13}$ It is based on a ternary component solvent system containing an extraction solvent, a dispersive solvent and an aqueous solution. ${ }^{11}$ DLLME presents advantages such as easy operation, low cost, rapidity, and high extraction efficiency. However, drawback associated with conventional DLLME is that toxic chlorinated solvents such as chloroform, carbon tetrachloride, chlorobenzenes are usually used as extraction solvent. ${ }^{14,15}$ These organic solvents may pose a potential threat to both human and the environment. Therefore, there is a growing demand for the development of environmentally friendly solvents to replace traditional highly toxic solvents. Recently, deep eutectic solvents (DESs), as a class of green solvents, have attracted considerable attention. ${ }^{16}$

DESs are liquid with a melting point much lower than either of the individual components. ${ }^{17-19}$ They are prepared by mixing hydrogen bond donors (HBDs) and hydrogen bond acceptors (HBAs). DESs share some physicochemical characteristics with ionic liquids (ILs) such as volatility, 
good thermal stability and tunable miscibility. ${ }^{20}$ Compared with ILs, DESs offer several unique properties, such as easy preparation, biodegradability and low cost. ${ }^{21-23}$ Therefore, DESs were used as extraction solvents for the extraction and preconcentration of different target analytes from various matrices. ${ }^{24}$ Generally, DESs used in DLLME are commonly mixtures of quaternary ammonium salts (trioctylmethylammonium chloride, choline chloride and tetrabutylammonium chloride) and different HBDs. ${ }^{25}$

Recently, considerable attention has been devoted to the development of hydrophobic DESs in sample preparation techniques. Hydrophobic DESs consisting of DL-menthol and aliphatic acids applied to alkali and metal ions removal from water have been reported. ${ }^{26}$ Later, DESs formed from DL-menthol with a variety of other chemical species (as HBDs) exhibited good results for the extraction and separation of various compounds. ${ }^{26-29}$ Hitherto, only small molecular chemicals, such as acetic acid, pyruvic acid, lactic acid, octanoic acid, decanoic acid and dodecanoic acid have been used as HBDs. Polyethylene glycol (PEG), as a colorless polyether polymer, is non-toxic and entirely biocompatible. Its green properties, various average molecular weights, excellent extraction properties and low cost made it a good candidate as HBD of DESs. ${ }^{30-32}$ As far as we know, DESs consisting of menthol and PEGs have not been investigated and used to extract any analytes from real samples.

Therefore, the aim of this study is to develop an easy and green method based on vortex-assisted DLLME (VA-DLLME) for the extraction and preconcentration of parabens. For this purpose, a new type of polymeric DES consisted of DL-menthol and PEGs including PEG 200, PEG 400 and PEG 600 at different molar ratios was prepared and used as the extraction solvents for the first time. Finally, the developed method was used to extract parabens in foods, cosmetics and pharmaceutical products prior to analysis with high-performance liquid chromatography couple to diode array detector (HPLC-DAD).

\section{Experimental}

\section{Chemicals and materials}

Methylparaben (MP) (98\%), ethylparaben (EP) (99\%), propylparaben (PP) (99\%), butylparaben (BP) (99\%), DL-menthol (98\%), PEG 200 (98\%), PEG 400 (99\%) and PEG 600 (99\%) were purchased from Aladdin Industrial Corporation (Shanghai, China). Merck was the supplier of HPLC grade methanol (Darmstadt, Germany). Ultrapure water was purified with a Milli-Q water purification system (Milford, MA, USA).
Stock solutions were prepared by dissolving the parabens in methanol at the concentration of $1 \mathrm{mg} \mathrm{mL}^{-1}$ and stored at $4{ }^{\circ} \mathrm{C}$ until use. The working solutions were obtained daily by diluting the stock solution to different concentrations in ultrapure water.

\section{Sample pretreatment}

In this study, commercial products including two foods (one orange juice and one peach juice), three cosmetics (two creams and one lotion) and two pharmaceutical products (two drug solutions, ophthalmic solution (drug solution 1) and lidocaine hydrochloride injection (drug solution 2) were used for extraction by the developed method. Foods and cosmetics were purchased from a supermarket and pharmaceutical products were obtained from a drug store in Kunming, China.

For cosmetics and pharmaceutical products, $50 \mathrm{mg}$ of each sample was mixed with $200 \mu \mathrm{L}$ of methanol by vortex mixing for $2 \mathrm{~min}$ and sonication for $30 \mathrm{~min}$. After dilution of the mixture with ultrapure water (100 times), the solution was vortexed and subject to centrifugation at $5000 \mathrm{rpm}$ for 5 min. Finally, the upper phase was collected and subjected to VA-DLLME procedure. Juices samples were centrifuged, and the supernatant was collected and diluted for 100 times as the experimental samples.

\section{Instrumentation}

The analysis of parabens was carried out on an Agilent (Palo Alto, CA, USA) 1260 Infinity HPLC system equipped with an auto-sampler, a quaternary pump, a thermostatted column compartment and a DAD. The chromatographic separation was performed on an Agilent Zorbax SB-C column $(150 \times 4.6 \mathrm{~mm}, 5 \mu \mathrm{m}$ particle). The injection volume was $10 \mu \mathrm{L}$ and the column temperature was $25{ }^{\circ} \mathrm{C}$. The mobile phase, composed of methanol (A) and ultrapure water (B), was eluted in a gradient mode

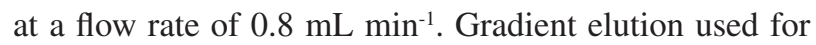
separation was: $0 \mathrm{~min}, 35 \%$ solvent $\mathrm{A}$; 0-10 min, 35-70\% solvent A; 10-17 min, 70\% solvent A; $17-20$ min, 70-35\% solvent $A$. The detection wavelength was set at $256 \mathrm{~nm}$. The identification of analyte is confirmed by the retention time. The structures of the prepared DESs were characterized by using a Varian Excalibur 3100 Fourier transform infrared (FTIR) spectroscopy (Varian, Palo Alto, USA) and INOVA-400 proton nuclear magnetic resonance ( ${ }^{1} \mathrm{H}$ NMR) spectrometer (Varian, Palo Alto, USA). FTIR spectra were obtained using $\mathrm{KBr}$ pellet technique. The ${ }^{1} \mathrm{H}$ NMR spectroscopy was conducted at ambient temperature by dilution with chloroform. 


\section{Synthesis and characterization of DESs}

In the present work, DESs composed of DL-menthol and PEG 200, PEG 400 or PEG 600 as HBDs at different molar ratios were prepared. The preparation procedure of a DES was as follows: mixing DL-menthol with HBD at a required molar ratio in a round-bottom flask at $70{ }^{\circ} \mathrm{C}$ with continuous magnetic stirring until the formation of a transparent liquid. The obtained DESs were cooled to room temperature and kept in desiccators until use.

\section{Extraction procedure}

To carry out VA-DLLME, $8 \mathrm{~mL}$ of the sample solution or paraben working solution was transferred into a $10 \mathrm{~mL}$ glass test tube with conical-bottom. Then, $100 \mu \mathrm{L}$ of DES was rapidly added to the sample solution, which was subsequently vortexed for $1 \mathrm{~min}$. When a cloudy solution containing fine droplets of DES appeared, the solution was subjected to centrifugation (5000 rpm, $4 \mathrm{~min}$ ). Consequently, the DES phase was found to be settled at the upper of the centrifuge tube. Finally, the aqueous phase was withdrawn with a syringe and the DES phase was collected and analyzed by HPLC-DAD.

\section{Calculation}

Enrichment factor (EF), defined as the ratio of the analyte concentration in the separated DES phase $\left(\mathrm{C}_{\mathrm{DES}}\right)$ to the initial concentration of analyte within aqueous phase $\left(\mathrm{C}_{\mathrm{aq}}\right)$, was determined according to the following equation:

$\mathrm{EF}=\frac{\mathrm{C}_{\mathrm{DES}}}{\mathrm{C}_{\mathrm{aq}}}$

Extraction recovery (ER, in percentage) was calculated by the following equation:

$\mathrm{ER}(\%)=\mathrm{EF} \times \frac{\mathrm{V}_{\mathrm{DES}}}{\mathrm{V}_{\mathrm{aq}}} \times 100$

$\mathrm{V}_{\mathrm{DES}}$ and $\mathrm{V}_{\mathrm{aq}}$ were DES volume and sample solution volume, respectively.

\section{Results and Discussion}

\section{Preparation and characterization of DESs}

In this study, DESs composed of DL-menthol and PEGs were successfully prepared as homogenous liquids without crystal precipitation. The prepared DESs were summarized in Table 1. The chemical structures of the prepared DESs were characterized by ${ }^{1} \mathrm{H}$ NMR and FTIR. The ${ }^{1} \mathrm{H}$ NMR spectrum and FTIR spectrum of representative DES-7 (used as extraction solvent) were investigated and summarized in Figures 1 and 2, respectively. As shown in Figure 1, the peaks of DES can be assigned to DL-menthol and PEG 400, and no additional peaks appeared.

Table 1. Preparation of different polymeric DES

\begin{tabular}{lccc}
\hline $\begin{array}{l}\text { Hydrogen } \\
\text { bond acceptor }\end{array}$ & $\begin{array}{c}\text { Hydrogen bond } \\
\text { donor }\end{array}$ & Molar ratio & Abbreviation \\
\hline & PEG 200 & $3: 1$ & DES-1 \\
& PEG 400 & $3: 1$ & DES-2 \\
& PEG 600 & $3: 1$ & DES-3 \\
& PEG 400 & $5: 1$ & DES-4 \\
DL-Menthol & PEG 400 & $4: 1$ & DES-5 \\
& PEG 400 & $2: 1$ & DES-6 \\
& PEG 400 & $1: 1$ & DES-7 \\
& PEG 400 & $1: 2$ & DES-8 \\
& PEG 400 & $1: 3$ & DES-9 \\
& PEG 400 & $1: 4$ & DES-10 \\
\hline
\end{tabular}

PEG: polyethylene glycol.

The formation of DESs is driven by the hydrogen bond between DL-menthol and PEG. To illustrate the interaction, FTIR spectra of DL-menthol, PEG 400 and DES-7 were tested. As seen in Figure 2, compared with the $\mathrm{O}-\mathrm{H}$ stretching vibration broad band at $3546 \mathrm{~cm}^{-1}$ of PEG 400, the $\mathrm{O}-\mathrm{H}$ stretching vibration broad band of DES shifted to $3230 \mathrm{~cm}^{-1}$. The phenomenon can conceivably be explained by the transfer of the oxygen atom cloud point to hydrogen bond lead to a slightly decrease in force constant, similar observations were reported in earlier paper as well. ${ }^{32}$ On the basis of the results, it can be concluded that the hydrogen bond appear between PEG 400 and DL-menthol and the DES was formed.

\section{Solubility of DES in water}

In DLLME, the immiscibility of DES with water is essential when it is applied to extract analytes from aqueous samples. According to previous work, ${ }^{33}$ the solubility behavior of the DESs in water was investigated by mixing a prepared DES and ultrapure water at a volume ratio of 1:3. Since PEG polymers can be able to form DESs with DL-menthol in wide molar ratio ranges, DESs composed of DL-menthol and PEG 400 from molar ratio of 5:1 to 


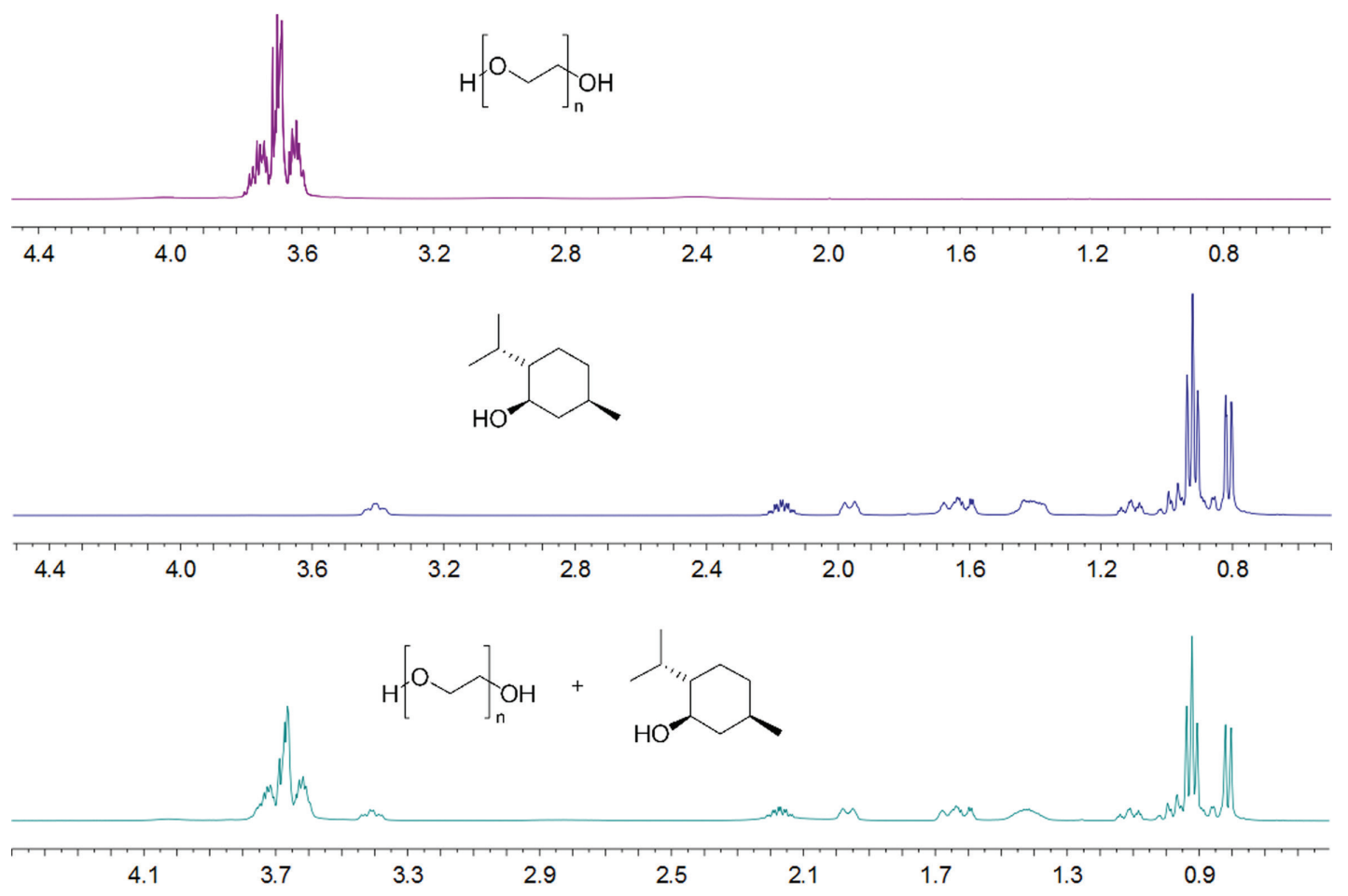

Figure 1. ${ }^{1} \mathrm{H}$ NMR spectra of PEG 400 (top); DL-menthol (middle) and DES-7 (bottom).

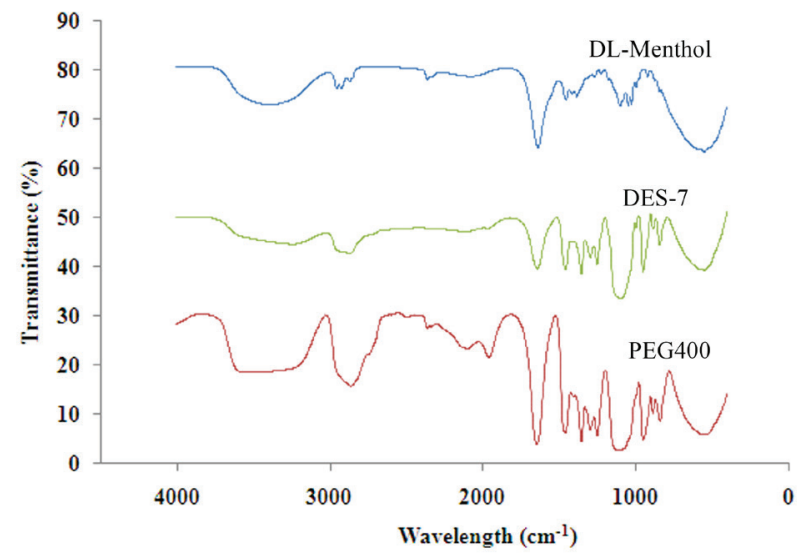

Figure 2. FTIR (KBr) spectra of DL-menthol, PEG 400 and DES-7.

$1: 4(5: 1,4: 1,3: 1,2: 1,1: 1,1: 2,1: 3$ and 1:4) were prepared and investigated. After vortex (30 s) and centrifugation (5000 rpm, $4 \mathrm{~min}$ ), it could be found that all the DESs could form two phases when water was added. Furthermore, the effect of average molecular weights of PEG on the DES solubility in water was investigated. Three DESs composed of DL-menthol and HBDs defined as PEG 200 (DES-1), 400 (DES-2) and 600 (DES-3) at a molar ratio of 3:1 were prepared. The ability of DESs to form two phases was in the following order: DES-3 > DES-2 > DES-1. The phenomenon can conceivably be explained by an increase of PEG average molecular weight led to enhancement of PEG hydrophobicity, similar observations were reported in earlier paper as well..$^{32}$

\section{Selection of extraction solvent}

The type of extraction solvent determines the extraction efficiency of VA-DLLME. In this study, three types of DES composed of DL-menthol and PEG200, 400 and 600 as the HBDs at a molar ratio of 3:1 were investigated. Figure 3a shows that DES-2 (DL-menthol and PEG 400) gave the highest chromatographic signals for the target analytes, as DES-2 has better affinity for parabens due to its suitable hydrophobicity. The molar ratio of HBA and HBD influences the extraction efficiency of DES, therefore, DES composed of DL-menthol and PEG 400 was used for further optimization of molar ratios.

\section{Effect of salt addition}

The salting-out effect can enhance the extraction efficiency in conventional liquid phase microextraction. In the present study, the solubility of analytes in the aqueous solution reduced with increasing ionic strength (salting-out effect). On the other hand, the volume of the DES phase raised with an increase of salt concentration, resulted in a decrease in the target analytes concentration. To investigate the salt addition effect on the extraction efficiency, different concentrations of $\mathrm{NaCl}(0-15 \%, \mathrm{~m} / \mathrm{v})$ were added to the aqueous solution. As shown in Figure 3b, the analytical signals initially increased a little when $1 \% \mathrm{NaCl}$ was added, and there were no significant changes thereafter. 

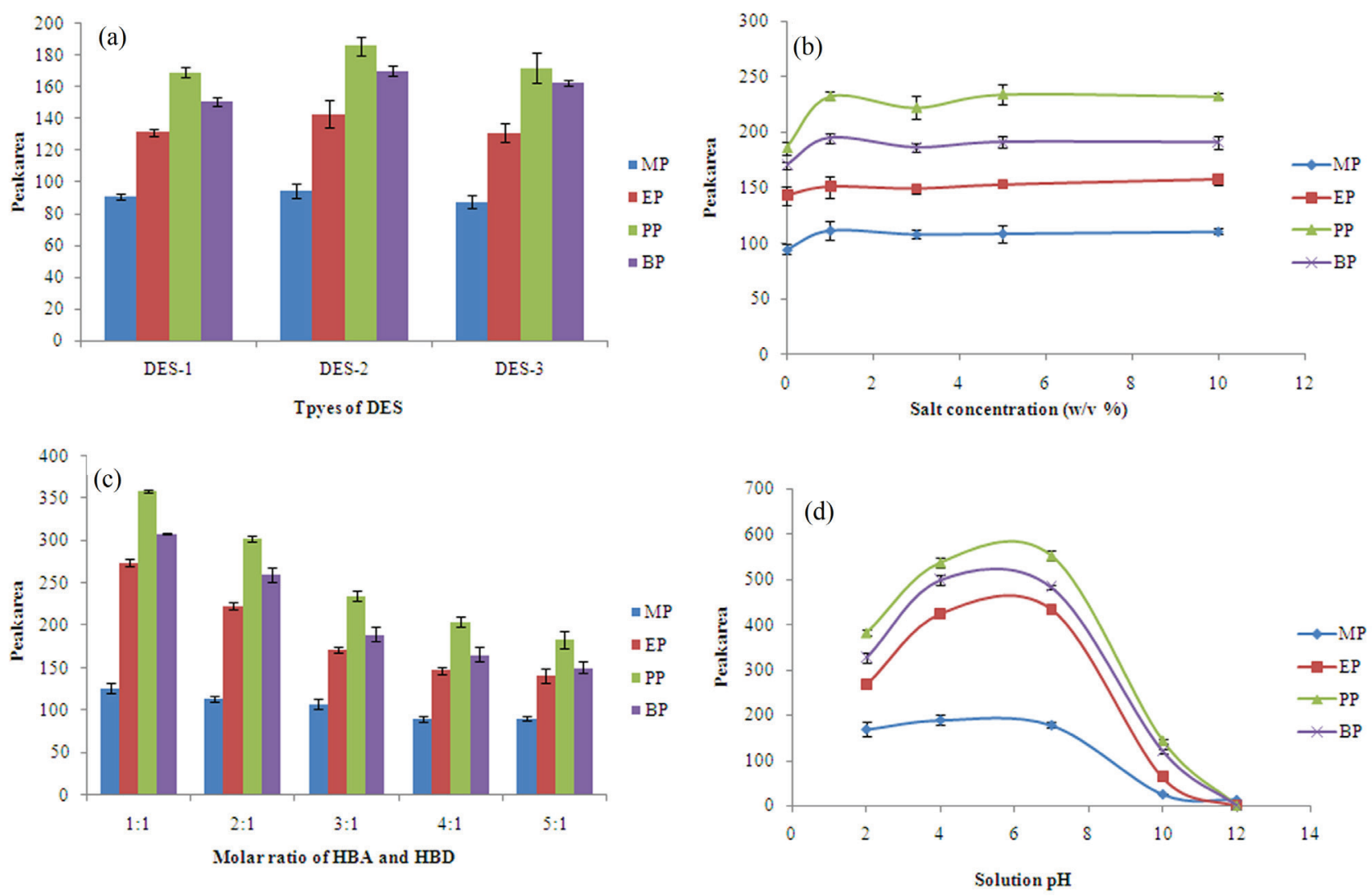

Figure 3. Optimization of the extraction efficiency of the DES-VA-DLLME process. (a) Effect of the type of DES on the extraction. (b) Effect of salt concentration on the extraction. (c) Effect of molar ratio of HBA and HBD on the extraction. (d) Effect of solution pH on the extraction. Three replicate experiments were conducted.

Therefore, further experiments were performed with $\mathrm{NaCl}$ concentration of $1 \%$.

\section{Effect of the composition of DES}

On the basis of preceding experiments, DES composed of DL-menthol and PEG 400 exhibited the best extraction efficiency for parabens. The molar ratio of HBA and HBD also affects the extraction efficiency of the DES in the present study. Hence, the extraction performance of DESs composed of DL-menthol and PEG 400 with different molar ratios (5:1, 4:1, 3:1, 2:1, 1:1, 1:2, 1:3 and 1:4) was explored. DESs with the molar ratios of 1:2, 1:3 and 1:4 were not convenient to recovery after the extraction, therefore, these DESs were not investigated. As shown in Figure 3c, DES-7 (at a molar ratio of 1:1) shows the best extraction ability for parabens. Therefore, DES-7 was chosen as the extraction solvent in this study.

\section{Effect of extraction solvent volume}

To investigate the effect of extraction solvent volume, different volumes of DES-7 (100, 120, 150, 180 and $200 \mu \mathrm{L}$ ) were tested. The peak areas decreased with the growing volume of extraction solvent (results are not shown). This might cause the decreased concentration of parabens in the DES phase due to the dilution effect. As a result, higher analytical signals were obtained at lower volumes of the DES-7. It should be noted that volumes lower than $100 \mu \mathrm{L}$ were not studied due to the difficulty in manipulation. Hence, $100 \mu \mathrm{L}$ of DES-7 were used for further experiments.

\section{Effect of vortex time}

VA-DLLME is a non-exhaustive process where time-dependent distribution is expected. The extraction efficiency depends on the partition of analytes between the sample and the DES. Vortex time in the range of 1-5 min was investigated. According to the obtained results, no significant change of chromatographic signals was observed with different extraction time (results are not shown). Therefore 1 min was employed as the vortex time.

\section{Effect of the aqueous phase $\mathrm{pH}$}

The solution $\mathrm{pH}$ also affects the migration of target analytes to the DES phase since it determines the existing 
form of the analytes. To evaluate the effect of solution $\mathrm{pH}$ on the extraction efficiency, $\mathrm{pH}$ values of the aqueous solutions were adjusted between 2 and 12 using $0.1 \mathrm{M} \mathrm{HCl}$ or $0.1 \mathrm{M} \mathrm{NaOH}$. As depicted in Figure 3d, the highest peak areas were obtained when the solution $\mathrm{pH}$ are in the range of 4 to 7, and significant decreases in peak areas were found at $\mathrm{pH} 10$ and 12. From the published studies, ${ }^{34,35}$ parabens are in their neutral form in the $\mathrm{pH}$ range of 4-7 since the $\mathrm{p} K_{\mathrm{a}}$ values of MP, EP, PP, and BP were $>8.2$, and the hydrolysis of the alcohol is observed when solution $\mathrm{pH}$ is higher than 8. In this study, $\mathrm{pH}$ of all aqueous solutions ranged between 4 and 7 , therefore, the solution $\mathrm{pH}$ was not adjusted in further experiments.

\section{Analytical features of the method}

After optimization of the relevant parameters, the proposed method was evaluated in terms of linear range of the calibration graphs, precision, limits of detection (LODs), limits of quantification (LOQs), EF and extraction recovery (ER, in percentage), and the results are summarized in Table 2. The linearity was investigated by extraction of spiked ultrapure water at seven different concentrations, varying between 1 and $1000 \mathrm{ng} \mathrm{mL}^{-1}$ for $\mathrm{BP}, 3$ and $1000 \mathrm{ng} \mathrm{mL}^{-1}$ for EP and PP, and 5 and $1000 \mathrm{ng} \mathrm{mL}^{-1}$ for MP. The regression coefficients (r) were in the range of
0.9995 and 0.9999 . Precision was calculated by extraction of aqueous samples at concentration levels of $10 \mathrm{ng} \mathrm{mL}^{-1}$. Relative standard deviations (RSDs) were $<4.71 \%$ for the experiments, indicating good precision of the method. LODs and LOQs (defined as the lowest concentrations yielding a signal-to-noise ( $\mathrm{S} / \mathrm{N})$ ratio of 3 and 10$)$ were in the range of 0.3-2 $\mathrm{ng} \mathrm{mL}^{-1}$ and $1-5 \mathrm{ng} \mathrm{mL}^{-1}$, respectively. The EFs and ER were calculated based on the equations 1 and 2 in sub-section "Calculation". EFs of 53-78 that correspondence to ER in the range of $66.3-97.5 \%$ were obtained.

\section{Application of the developed methods to real samples}

To verify the applicability of the developed method, two fruit juice samples, three cosmetic and two pharmaceutical products were analyzed under the optimized conditions. The detected concentrations of parabens were recorded as $\mathrm{mg} \mathrm{g}^{-1}$. As shown in Table 3, parabens were not detected in two juices samples, and they were found at $0.68-1.49 \mathrm{mg} \mathrm{g}^{-1}$ in cosmetics and pharmaceutical samples, expressed as the mean value $(n=3)$. To evaluate the sample matrix effect, recovery experiments were conducted by spiking standard solutions of parabens at concentration levels of 10 and $100 \mathrm{ng} \mathrm{mL}^{-1}$ into real sample solutions. The recovery was defined as the ratio of peak areas of the spiked real sample extracts to spiked ultrapure water extracts. The

Table 2. Quantitative features of the developed method

\begin{tabular}{lccccccc}
\hline Analyte & Linearity / $(\mathrm{ng} \mathrm{mL}-1)$ & $\mathrm{r}$ & $\mathrm{LOD} /(\mathrm{ng} \mathrm{mL}-1)$ & $\mathrm{LOQ} /\left(\mathrm{ng} \mathrm{mL} \mathrm{m}^{-1}\right)$ & $\mathrm{RSDs} / \%$ & $\mathrm{EF}$ & $\mathrm{ER} / \%$ \\
\hline MP & $5-1000$ & 0.9995 & 2 & 5 & 4.71 & 53 & 66.3 \\
EP & $3-1000$ & 0.9997 & 1 & 3 & 1.33 & 73 & 91.2 \\
PP & $3-1000$ & 0.9999 & 1 & 3 & 2.69 & 78 & 97.5 \\
BP & $1-1000$ & 0.9996 & 0.3 & 1 & 2.91 & 77 & 96.3 \\
\hline
\end{tabular}

r: regression coefficient; LOD: limit of detection; LOQ: limit of quantification; RSD: relative standard deviation; EF: enrichment factor; ER: extraction recovery; MP: methylparaben; EP: ethylparaben; PP: propylparaben; BP: butylparaben.

Table 3. Analytical results of the four parabens in real samples

\begin{tabular}{|c|c|c|c|c|c|c|c|c|}
\hline \multirow{2}{*}{$\begin{array}{l}\text { Analyte } \\
\text { Sample }\end{array}$} & \multicolumn{2}{|c|}{ MP } & \multicolumn{2}{|c|}{ EP } & \multicolumn{2}{|c|}{ PP } & \multicolumn{2}{|c|}{ BP } \\
\hline & $\begin{array}{c}\text { Detected } \\
\text { amount / } \\
\left(\mathrm{mg} \mathrm{g}^{-1}\right)\end{array}$ & RSD / \% & $\begin{array}{c}\text { Detected } \\
\text { amount / } \\
\left(\mathrm{mg} \mathrm{g}^{-1}\right)\end{array}$ & RSD / \% & $\begin{array}{c}\text { Detected } \\
\text { amount / } \\
\left(\mathrm{mg} \mathrm{g}^{-1}\right)\end{array}$ & RSD / \% & $\begin{array}{c}\text { Detected } \\
\text { amount / } \\
\left(\mathrm{mg} \mathrm{g}^{-1}\right)\end{array}$ & $\mathrm{RSD} / \%$ \\
\hline Hand cream & 0.68 & 4.79 & 1.40 & 5.01 & nd & & nd & \\
\hline Face cream & 1.49 & 2.14 & nd & & 0.92 & 2.30 & nd & \\
\hline Lotion & 1.26 & & nd & & nd & & nd & \\
\hline Drug solution 1 & nd & & 0.70 & 1.27 & nd & & nd & \\
\hline Drug solution 2 & nd & & 0.85 & 1.71 & nd & & nd & \\
\hline Orange juice & nd & & nd & & nd & & nd & \\
\hline Peach juice & nd & & nd & & nd & & nd & \\
\hline
\end{tabular}

nd: not detected; RSD: relative standard deviation; MP: methylparaben; EP: ethylparaben; PP: propylparaben; BP: butylparaben. 
Table 4. Results of experiments to investigate the sample matrix effect for the parabens

\begin{tabular}{|c|c|c|c|c|c|c|c|c|}
\hline \multirow[b]{2}{*}{ Sample } & \multicolumn{2}{|c|}{ MP } & \multicolumn{2}{|c|}{ EP } & \multicolumn{2}{|c|}{ PP } & \multicolumn{2}{|c|}{$\mathrm{BP}$} \\
\hline & $\begin{array}{c}\text { Added / } \\
\left(\mathrm{ng} \mathrm{mL}^{-1}\right)\end{array}$ & $\begin{array}{c}\text { Recovery } \pm \\
\text { RSD / \% }\end{array}$ & $\begin{array}{c}\text { Added / } \\
\left(\mathrm{ng} \mathrm{mL}^{-1}\right)\end{array}$ & $\begin{array}{c}\text { Recovery } \pm \\
\text { RSD / \% }\end{array}$ & $\begin{array}{c}\text { Added / } \\
\left(\text { ng mL }^{-1}\right)\end{array}$ & $\begin{array}{c}\text { Recovery } \pm \\
\text { RSD / \% }\end{array}$ & $\begin{array}{c}\text { Added / } \\
\left(\mathrm{ng} \mathrm{mL}^{-1}\right)\end{array}$ & $\begin{array}{c}\text { Recovery } \pm \\
\text { RSD / \% }\end{array}$ \\
\hline \multirow{2}{*}{ Orange juice } & 10 & $95.7 \pm 6.2$ & 10 & $89.8 \pm 1.4$ & 10 & $100.1 \pm 2.5$ & 10 & $100.8 \pm 3.6$ \\
\hline & 100 & $97.2 \pm 1.6$ & 100 & $94.7 \pm 3.8$ & 100 & $90.2 \pm 1.4$ & 100 & $92.2 \pm 4.8$ \\
\hline \multirow{2}{*}{ Peach juice } & 10 & $100.9 \pm 2.3$ & 10 & $86.7 \pm 5.1$ & 10 & $99.6 \pm 2.3$ & 10 & $90.1 \pm 2.8$ \\
\hline & 100 & $98.2 \pm 3.5$ & 100 & $109.1 \pm 2.6$ & 100 & $89.1 \pm 4.5$ & 100 & $102.4 \pm 1.7$ \\
\hline \multirow{2}{*}{ Hand cream } & 10 & $91.1 \pm 6.8$ & 10 & $103.3 \pm 1.1$ & 10 & $93.1 \pm 3.2$ & 10 & $93.5 \pm 4.1$ \\
\hline & 100 & $98.0 \pm 4.4$ & 100 & $89.1 \pm 3.5$ & 100 & $99.4 \pm 5.7$ & 100 & $102.6 \pm 0.6$ \\
\hline \multirow{2}{*}{ Face cream } & 10 & $98.3 \pm 5.3$ & 10 & $94.7 \pm 3.1$ & 10 & $98.8 \pm 1.9$ & 10 & $95.2 \pm 3.1$ \\
\hline & 100 & $92.6 \pm 4.1$ & 100 & $94.6 \pm 4.6$ & 100 & $94.7 \pm 5.0$ & 100 & $107.8 \pm 1.9$ \\
\hline \multirow{2}{*}{ Toner } & 10 & $94.6 \pm 0.5$ & 10 & $94.9 \pm 4.1$ & 10 & $103.6 \pm 0.9$ & 10 & $98.8 \pm 2.6$ \\
\hline & 100 & $92.0 \pm 1.0$ & 100 & $104.5 \pm 0.9$ & 100 & $96.8 \pm 1.4$ & 100 & $94.8 \pm 3.6$ \\
\hline \multirow{2}{*}{ Drug solution 1} & 10 & $105.1 \pm 2.2$ & 10 & $100.1 \pm 3.5$ & 10 & $92.8 \pm 4.9$ & 10 & $97.4 \pm 1.2$ \\
\hline & 100 & $94.9 \pm 7.3$ & 100 & $90.6 \pm 6.4$ & 100 & $104.3 \pm 7.7$ & 100 & $92.4 \pm 2.6$ \\
\hline \multirow{2}{*}{ Drug solution 2} & 10 & $87.9 \pm 3.9$ & 10 & $99.7 \pm 1.8$ & 10 & $99.8 \pm 0.6$ & 10 & $104.5 \pm 6.7$ \\
\hline & 100 & $91.9 \pm 2.6$ & 100 & $95.1 \pm 4.5$ & 100 & $101.2 \pm 4.9$ & 100 & $100.7 \pm 0.9$ \\
\hline
\end{tabular}

RSD: relative standard deviation; MP: methylparaben; EP: ethylparaben; PP: propylparaben; BP: butylparaben.

representative chromatogram for the determination of parabens in orange juice is shown in Figure 4. As can be seen in Table 4, recoveries of the four parabens ranged from 86.7 to $109.1 \%$ with RSD of $0.5-7.7 \%$. These results demonstrated that the present method is free from the interference of the matrix and feasible for the determination of parabens in real samples.

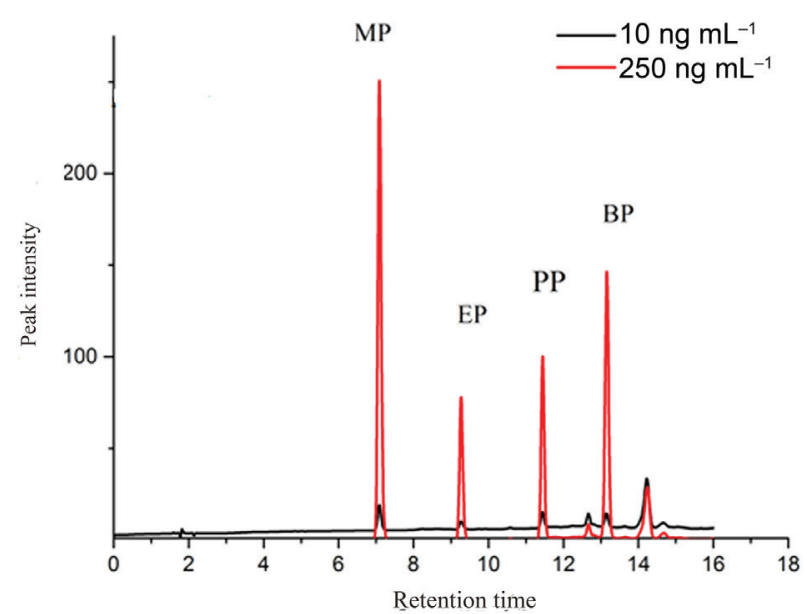

Figure 4. Representative chromatogram for the determination of parabens in orange juice.

\section{Comparison of the developed method with other methods}

The analytical features of the proposed method were compared with previously reported methods. ${ }^{1,11,35-38}$ As the results summarized in Table 5, the developed method is effective for the determination of parabens, with LODs better than those of methods 1-4, and comparable with methods 5 and 6 (with the much more sensitive detector). Regarding to the extraction time, conventional methods required 10-50 $\mathrm{min}$, while the developed method was shortened to $5 \mathrm{~min}$. Moreover, the proposed method presents similar precision to those of the reported methods. Therefore, the developed method can be applied to determine parabens in foods, cosmetics and pharmaceutical products with good accuracy, precision and fast extraction.

\section{Conclusions}

In the present study, DESs composed of DL-menthol and PEGs (with average molecular weights of 200, 400 and 600) were prepared successfully and used as extraction solvents for the DLLME of parabens for the first time. The DESs were easy to prepare and provided high extraction efficiency. Under the optimized conditions, the developed method showed good precision, accuracy and reproducibility. The developed method was applied for the extraction of four parabens in foods, cosmetics and pharmaceutical products, and good recoveries in a range of $87.9-107.8 \%$ were obtained. The main advantage of the proposed method is that DES, which is called as a green extraction solvent, can reduce the cost of routine analysis and environmental 
Table 5. Comparison of the proposed method with other methods in determination of the parabens

\begin{tabular}{|c|c|c|c|c|c|c|c|}
\hline Method & $\begin{array}{l}\text { Method } \\
\text { No. }\end{array}$ & $\begin{array}{l}\text { Extraction } \\
\text { time / min }\end{array}$ & $\mathrm{RSD} / \%$ & $\begin{array}{l}\text { Linearity / } \\
\left(\mathrm{ng} \mathrm{mL} \mathrm{mL}^{-1}\right)\end{array}$ & $\begin{array}{c}\mathrm{LOD} / \\
\left(\mathrm{ng} \mathrm{mL}^{-1}\right)\end{array}$ & Sample & Reference \\
\hline DLLME-GC-FID $^{a}$ & 1 & 10 & $2-3$ & $20-30000$ & $5-15$ & $\begin{array}{l}\text { hygienic product, pharmaceuticals } \\
\text { and food samples }\end{array}$ & 35 \\
\hline VA-DLLME-SFO-CapLC-UV & 2 & 5.5 & $0.33-4.57$ & $100-10000$ & $10-30$ & $\begin{array}{l}\text { food products, cosmetic products } \\
\text { and pharmaceuticals }\end{array}$ & 1 \\
\hline DLLME-CE-UV ${ }^{c}$ & 3 & 1 & $0.6-3.3$ & $300-6000$ & $100-200$ & pharmaceuticals & 11 \\
\hline SPME-HPLC-UV ${ }^{\mathrm{d}}$ & 4 & 23 & $3.3-6.9$ & $500-160000$ & $120-150$ & cosmetic samples & 36 \\
\hline SM-SLLME-LC-MS-MS ${ }^{\mathrm{e}}$ & 5 & 45 & $1.1-8.0$ & $0.40-100$ & $0.10-0.20$ & human breast milk samples & 37 \\
\hline LLE-LC-MS-MS ${ }^{\mathrm{f}}$ & 6 & $>70$ & $5.8-18.6$ & $0.5-100$ & $0.5-1$ & urine & 38 \\
\hline DLLME-HPLC-DAD & & 5 & $1.33-4.71$ & $1-1000$ & $0.3-2$ & $\begin{array}{l}\text { food samples, pharmaceuticals } \\
\text { and cosmetics products }\end{array}$ & this study \\
\hline
\end{tabular}

${ }^{a}$ Dispersive liquid-liquid microextraction coupled with gas chromatography-flame ionization detector; ${ }^{b}$ vortex-assisted dispersive liquid-liquid microextraction based on the solidification of a floating organic drop coupled with capillary liquid chromatography-UV detection; 'dispersive liquid-liquid microextraction coupled with capillary electrophoresis-UV detection; ${ }^{\mathrm{d}}$ solid phase microextraction coupled with high performance liquid chromatographyUV detection; ${ }^{e}$ stir-membrane solid-liquid-liquid microextraction coupled with ultra-high performance liquid chromatography-tandem mass spectrometry; fliquid-liquid extraction coupled with liquid chromatography-tandem mass spectrometry. RSD: relative standard deviation; LOD: limit of detection; DLLME-HPLC-DAD: dispersive liquid-liquid microextraction with high-performance liquid chromatography couple to diode array detector.

burden. Besides parabens, the polymeric-based DESs can be regarded as promising solvents for the extraction of other trace organic compounds in real samples.

\section{Acknowledgments}

The authors acknowledge the financial support of Yunnan key laboratory of food-safety testing technology and Yunnan Local Colleges Applied Basic Research Projects (2018FH001-022).

\section{References}

1. Chen, C.-W.; Hsu, W.-C.; Lu, Y.-C.; Weng, J.-R.; Feng, C.-H.; Food Chem. 2018, 241, 411.

2. Makkliang, F.; Kanatharana, P.; Thavarungkul, P.; ThammakhetBuranachai, C.; Talanta 2018, 184, 429.

3. Darbre, P. D.; Byford, J. R.; Shaw, L. E.; Horton, R. A.; Pope, G. S.; Sauer, M. J.; J. Appl. Toxicol. 2002, 22, 219.

4. Darbre, P. D.; Harvey, P. W.; J. Appl. Toxicol. 2008, 28, 561.

5. Okubo, T.; Yokoyama, Y.; Kano, K.; Kano, I.; Food Chem. Toxicol. 2001, 39, 1225.

6. European Union (EU); Council Directive 76/768/EEC on the Approximation of The Laws of the Member States Relating to Cosmetic Products; Official Journal of the European Union L 262, 27/07/1976, p. 169.

7. Hashemi, B.; Zohrabi, P.; Kim, K.-H.; Shamsipur, M.; Deep, A.; Hong, J.; TrAC, Trends Anal. Chem. 2017, 97, 83.

8. Filik, H.; Avan, A. A.; Food Chem. 2018, 242, 301.

9. Mirmahdieh, M. S. S.; J. Sep. Sci. 2009, 32, 988.

10. Chatzimitakos, T.; Binellas, C.; Maidatsi, K.; Stalikas, C.; Anal. Chim. Acta 2016, 910, 53.
11. Alshana, U.; Ertaş, N.; Göğer, N. G.; Food Chem. 2015, $181,1$.

12. Zhou, H.-T.; Ding, E. M. C.; Ding, W.-H.; J. Chromatogr. B 2017, 1058, 14.

13. Rezaee, M.; Assadi, Y.; Hosseini, M. R. M.; Aghaee, E.; Ahmadi, F.; Berijani, S.; J. Chromatogr. A 2006, 1116, 1.

14. Petrarca, M. H.; Godoy, H. T.; Food Chem. 2018, 257, 44.

15. Rykowska, I.; Ziemblińska, J.; Nowak, I.; J. Mol. Liq. 2018, 259, 319.

16. Shishov, A.; Bulatov, A.; Locatelli, M.; Carradori, S.; Andruch, V.; Microchem. J. 2017, 135, 33.

17. Abbott, A. P.; Capper, G.; Davies, D. L.; Rasheed, R. K.; Tambyrajah, V.; Chem. Commun. 2003, 7, 70.

18. Vanda, H.; Dai, Y.; Wilson, E. G.; Verpoorte, R.; Choi, Y. H.; C. R. Chim. 2018, 21, 628.

19. Zhang, Q.; Vigier, K. D. O.; Royer, S.; Jérôme F.; Chem. Soc. Rev. 2012, 41, 7108.

20. Yu, Y.; Lu, X.; Zhou, Q.; Dong, K.; Yao, H.; Zhang, S.; Chem. - Eur. J. 2008, 14, 11174.

21. Altamash, T.; Atilhan, M.; Aliyan, A.; Ullah, R.; Nasser, M.; Aparicio, S.; Chem. Eng. Technol. 2017, 40, 778.

22. Durand, E.; Lecomte, J.; Villeneuve, P.; Eur. J. Lipid Sci. Technol. 2013, 115, 379.

23. Weaver, K. D.; Kim, H. J.; Sun, J.; MacFarlane, D. R.; Elliott, G. D.; Green Chem. 2010, 12, 507.

24. Cunla, S. C.; Fermandes, J.; TrAC, Trends Anal. Chem. 2018, 105, 225.

25. Makoś, P.; Słupek, E.; Gębicki, J.; Microchem. J. 2020, 152, 104384.

26. van Osch, D. J. G. P.; Parmentier, D.; Dietz, C. H. J. T.; van den Bruinhorst, A.; Tuinier, R.; Kroon, M. C.; Chem. Commun. 2016, 52, 11987. 
27. Florindo, C.; Branco, L. C.; Marrucho, I. M.; Fluid Phase Equilib. 2017, 448, 135.

28. Kř́̌žek, T.; Bursová, M.; Horsley, R.; Kuchař, M.; Hložek, T.; J. Cleaner Prod. 2018, 193, 391.

29. Zarei, A. R.; Nedaei, M.; Ghorbanian, S. A.; J. Chromatogr. A 2018, 1553, 32.

30. Hou, X.; Yu, H.; Guo, Y.; Liang, X.; Wang, S.; Wang, L.; Liu, X.; J. Sep. Sci. 2015, 38, 2700.

31. Rahma, W. S. A.; Mjalli, F. S.; Al-Wahaibi, T.; Al-Hashmi, A. A.; Chem. Eng. Res. Des. 2017, 120, 271.

32. Zhang, H.; Wang, Y.; Zhou, Y.; Xu, K.; Li, N.; Wen, Q.; Yang, Q.; Talanta 2017, 170, 266.

33. Deng, W.; Yu, L.; Li, X.; Chen, J.; Wang, X.; Deng, Z.; Xiao, Y.; Food Chem. 2019, 274, 891.
34. Çabuk, H.; Akyüz, M.; Ata, Ş.; J. Sep. Sci. 2012, 35, 2645.

35. Farajzadeh, M. A.; Djozan, D.; Bakhtiyari, R. F.; Talanta 2010 , 81,1360

36. Fei, T.; Li, H.; Ding, M.; Ito, M.; Lin, J.-M.; J. Sep. Sci. 2011, 34, 1599.

37. Rodriguez-Gomez, R.; Roldan-Pijuan, M.; Lucena, R.; Cardenas, S.; Zafra-Gomez, A.; Ballesteros, O.; Navalon, A.; Valcarcel, M.; J. Chromatogr. A 2014, 1324, 26.

38. Asimakopoulos, A. G.; Wang, L.; Thomaidis, N. S.; Kannan, K.; J. Chromatogr. A 2014, 1324, 141.

Submitted: February 16, 2020

Published online: June 12, 2020 